\title{
DESEMPENHO PRODUTIVO DE VACAS GIROLANDO ESTIMADO PELO MODELO DE WOOD AJUSTADO POR METODOLOGIA BAYESIANA
}

\author{
(Productive performance of cow Girolando estimated by Wood's model \\ adjusted by bayesian methodology)
Laís Aberrachid Jacopini ${ }^{1}$, Severino Benone Paes Barbosa, Daniela Andressa Lino Lourenço, Marcos Vinícius Gualberto Barbosa da Silva

\begin{abstract}
${ }^{1}$ Correspondência: lais-jacopini@hotmail.com
RESUMO: Utilizou-se o modelo de Wood analisado por metodologia bayesiana, para estimar os componentes da curva de lactação de diferentes grupos genéticos, de primeira (OP1) e segunda (OP2) ordem de parto: 1/4 Holandês - 3/4 Gir; 1/2 Holandês - 1/2 Gir; 3/4 Holandês - 1/4 Gir; 5/8 Holandês - 3/8 Gir e 7/8 Holandês - 1/8 Gir. Os dados de produção de leite até 305 dias de lactação pertencem ao Arquivo Zootécnico do Centro Nacional de Pesquisa em Gado de Leite da Empresa Brasileira de Pesquisa Agropecuária (EMBRAPA/CNPGL). Após a estimação dos parâmetros do modelo, foram calculadas produção no pico (PP), tempo para atingir o pico (TP), persistência na lactação (PER) e duração da lactação para cada grupo genético. Animais de OP1 apresentaram menores produções por todas as fases da lactação, além de demorarem mais tempo para alcançar o pico de produção. Animais de OP2 apresentaram maior produção total de leite. A produção total de leite dos animais $7 / 8 \mathrm{H}-1 / 8 \mathrm{G}$ de $\mathrm{OP} 1$ foi semelhante a dos animais $3 / 4 \mathrm{H}-1 / 4 \mathrm{G}$ e $1 / 2 \mathrm{H}-1 / 2 \mathrm{G}$. Para a $\mathrm{OP} 2$, os animais mais produtivos foram os $7 / 8 \mathrm{H}-1 / 8 \mathrm{G}$, seguidos dos $3 / 4 \mathrm{H}-1 / 4 \mathrm{G}$ e $1 / 2 \mathrm{H}-1 / 2 \mathrm{G}$. Produção intermediaria foi verificada nos animais $5 / 8 \mathrm{H}-3 / 8 \mathrm{G}$ e inferiores nos animais $1 / 4 \mathrm{H}-3 / 4 \mathrm{G}$ para ambas as ordens de parto. Não foi observada diferença significativa para duração da lactação entre os grupos genéticos estudados. Animais de OP1 são mais persistentes que animais de OP2, porém, estes são mais produtivos. Os grupos genéticos estudados se mostram persistentes quanto à duração da lactação. A metodologia bayesiana no ajuste do modelo de Wood permite estimativas acuradas das características da curva de lactação dos animais e na estimação de produção total de leite, duração da lactação e persistência.
\end{abstract}

Palavras-chave: curva de lactação; modelo não linear; persistência na lactação; produção de leite

\begin{abstract}
It were used the model of Wood analyzed by bayesian methodology to estimates the components of the lactation curve of different genetic groups, first (OP1) and second (OP2) order of birth: 1/4 Holstein - 3/4 Gir, 1/2 Holstein - 1/2 Gir, 3/4 Holstein - 1/4 Gir, 5/8 Holstein - 3/8 Gir and 7/8 Holstein - 1/8 Gir. Data for milk production to 305 days of lactation belongs to the Animal Science Archive Research Center National Dairy Cattle from the Brazilian Agricultural Research Corporation (EMBRAPA / CNPGL). After estimating the model parameters were calculated the peak production (PP), time at peak (TP), persistency of lactation (PER) and lactation length for each genetic group. OP1 animals had lower yields for all stages of lactation, and take longer to reach peak production. OP2 animals had higher total milk production. The total milk production of animals $7 / 8 \mathrm{H}-1 / 8 \mathrm{G}$ of $\mathrm{OP} 1$ was similar in animals $3 / 4 \mathrm{H}-1 / 4 \mathrm{G} 1 / 2 \mathrm{H}-1 / 2 \mathrm{G}$. For $\mathrm{OP} 2$, the animals $7 / 8 \mathrm{H}-1 / 8 \mathrm{G}$ were the most productive, followed by $3 / 4 \mathrm{H}-1 / 4 \mathrm{G}$ and $1 / 2 \mathrm{H}-1 / 2 \mathrm{G}$. Intermediate production was observed in animals $5 / 8 \mathrm{H}-3 / 8 \mathrm{G}$ and lower in animals $1 / 4 \mathrm{H}-3 / 4 \mathrm{G}$ for both orders of birth. There was no significant difference in lactation length among genetic groups. OP1 animals are more persistent than OP2 animals, however, they are most productive. The genetic groups studied are shown similar for the duration of lactation. The bayesian approach in adjusting the Wood's model allows accurate estimates lactation curve characteristics of the animals and the estimation of total milk yield, lactation length and persistence.
\end{abstract}

Key Words: lactation curve; lactation persistence; milk yield nonlinear model 


\section{INTRODUÇÃO}

Devido à grande extensão territorial e diversidade climática a produção de leite no Brasil caracterizase principalmente por animais cruzados, com objetivo da expressão da heterose e da complementariedade entre as raças, buscando obter animais mais adaptados e produtivos nas condições do País. O principal cruzamento com esta finalidade é entre as raças Holandesa e Gir.

A produção de leite de um animal por um período de tempo caracteriza a curva de lactação, que pode ser influenciada além da questão genética, por fatores de ambiente. Com a curva de lactação é possível acompanhar o desempenho produtivo dos animais, facilitando técnicas de manejo, alimentação e descarte dos animais de acordo com o padrão produtivo desejável, além de avaliar os reprodutores antecipadamente com base nas lactações incompletas de suas filhas.

A curva de lactação normalmente apresenta três fases: produção inicial, taxa de acréscimo até 0 pico de produção e taxa de declínio após o pico. O pico de produção é definido como a produção máxima de leite alcançada em um dia de lactação, sendo um dos principais fatores que determinam a produção de leite, o período e a forma da curva de lactação (WOOD, 1967). A persistência na lactação é outro fator importante a ser considerado na curva de lactação, sendo definida como a capacidade da vaca em manter sua produção de leite após ter atingido produção máxima na lactação (COBUCl et al., 2004) e é considerada o principal componente da curva (WOOD, 1967). Neste sentido, tem-se que a produção total de leite é função da persistência, do pico de produção de leite e da duração da lactação (LUDWICK \& PETERSEN, 1943).

A curva de lactação se mostra importante por auxiliar na caracterização da produção animal durante toda a lactação, podendo ser identificados o tempo de ascensão ao pico, o pico de produção, a persistência na produção, a duração da lactação, além das quedas bruscas de produção e resposta a dietas e manejo (REKAYA et al., 2000).

Objetivou-se avaliar 0 desempenho produtivo, a duração da lactação, o tempo para atingir o pico, a produção de leite no pico e a persistência de vacas Girolando, de diferentes composições genéticas, por meio dos parâmetros da curva de lactação do modelo de Wood (1967) ajustado por abordagem Bayesiana. 


\section{MATERIAL E MÉTODOS}

Foram utilizados dados de produção de leite de 1.693 vacas de primeira (1 $1^{\text {a lact})}$ e 2.951 vacas de segunda (2a lact) lactação, totalizando 41.050 observações com partos ocorridos entre os anos 1991 a 2010, pertencentes ao Arquivo Zootécnico do Centro Nacional de Pesquisa em Gado de Leite, da Empresa Brasileira de Pesquisa Agropecuária (EMBRAPA/CNPGL). Os animais eram provenientes do cruzamento entre as raças Holandesa $(H)$ e Gir $(G)$, com diferentes composições genéticas: $1 / 4 \mathrm{H}$ 3/4G; 1/2H-1/2G; 3/4H-1/4G; 5/8H-3/8G e $7 / 8 \mathrm{H}-1 / 8 \mathrm{G}$. Aproximadamente $80 \%$ dos dados eram provenientes de rebanhos de Minas Gerais, Goiás e São Paulo. $O$ número de animais e observações para cada grupo genético pode ser visualizado na Tabela 1.

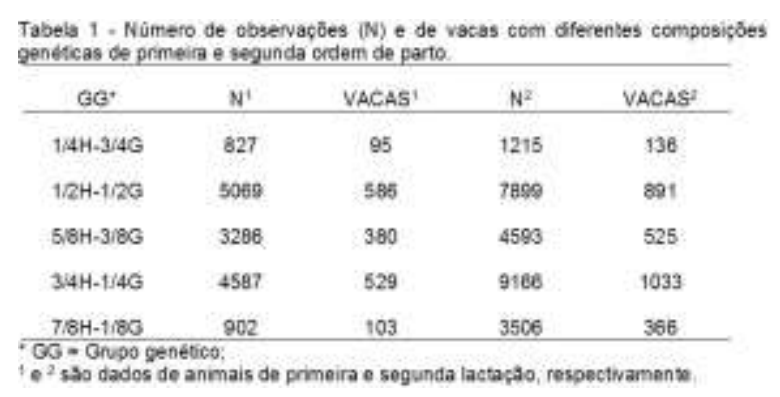

Foram eliminadas informações de animais que não possuíam data de nascimento, data de parto e data de encerramento da lactação. Foram consideradas as informações de lactações completas (até 305 dias) e que tivessem de oito a 10 controles por lactação.

Para estimar a curva de lactação dos animais utilizou-se o modelo de Wood (1967): $Y=a n^{b} e^{-c n}$, em que: $Y$ é a produção de leite $(\mathrm{kg})$ ao tempo $n$ de lactação; a é o parâmetro associado à produção diária no início da lactação; $b$ é o parâmetro que mede a inclinação da fase ascendente até o pico de produção; $c$ é o parâmetro referente à inclinação da fase descendente após o pico de produção e e é exponencial.

A análise dos modelos foi realizada através do software $R$ (2012). Considerou-se prioris não informativas para os parâmetros. A distribuição marginal a posteriori para os parâmetros foi obtida utilizando-se o pacote BRugs. Para todos os parâmetros foram geradas cadeias de 100.000 iterações, considerando um período de descarte inicial de 10.000 valores, sendo as amostras retiradas a cada 10 iterações. A análise de convergência das cadeias foi verificada utilizando-se o critério de Heidelberger \& Welch (1983), disponível no pacote CODA. Através dos parâmetros do modelo ajustado foram também calculados o tempo para atingir o pico $(P)$, a produção de leite no pico $(P P)$ e a persistência, em que $P=b / c$ e 
$\mathrm{PP}=\mathrm{a}[(\mathrm{b} / \mathrm{c}) \mathrm{b}] \mathrm{e}^{-\mathrm{b}} ; \quad$ e $\quad \mathrm{PER}=[-(\mathrm{b}+1) \ln (\mathrm{c})]$, segundo Wood (1967).

\section{RESULTADOS E DISCUSSÃO}

As estimativas dos parâmetros do modelo de Wood (1967) ajustado por metodologia bayesiana para os animais com diferentes composições genéticas e ambas as ordens de parto podem ser visualizadas na Tabela 2. Constam também os valores de coeficiente de determinação ajustado $\left(\mathrm{R}^{2} \mathrm{~A}\right)$ para cada grupo genético.

\begin{tabular}{|c|c|c|c|c|}
\hline \multirow{3}{*}{ Grupo genético } & \multicolumn{3}{|c|}{ Parâmetros do modelo } & \multirow{2}{*}{$R^{2} /(7)$} \\
\hline & a & $b$ & c & \\
\hline & \multicolumn{4}{|c|}{$1^{2}$ lactaçalo } \\
\hline $1 / 4 H-3 / 4 G$ & 8.27 & 0,1071 & 0,002890 & 37,76 \\
\hline $1 / 2 \mathrm{H}-1 / 2 \mathrm{G}$ & 11,26 & 0,1424 & 0,003170 & 82,81 \\
\hline $5 / 8 H-3 / 80$ & 11,19 & 0,1204 & 0,003124 & 75,38 \\
\hline $3 / 4 \mathrm{H}-1 / 4 \mathrm{G}$ & 10.89 & 0,1562 & 0,003158 & 78,59 \\
\hline \multirow[t]{2}{*}{ 7/BH-1/8G } & 13,30 & 0,1643 & 0,003356 & 53,15 \\
\hline & \multicolumn{4}{|c|}{$z^{\circ}$ lactafalo } \\
\hline $1 / 4 H-3 / 4 G$ & 11.22 & 0,1010 & 0,002949 & 61,98 \\
\hline $1 / 2 H-1 / 2 \mathrm{G}$ & 14,05 & 0,1165 & 0,003351 & 91,03 \\
\hline 5/EH-3/8G & 12,67 & 0,1436 & 0,003818 & 89,34 \\
\hline $3 / 4 \mathrm{H}-1 / 4 \mathrm{G}$ & 14,49 & 0,1259 & 0,003358 & 80,71 \\
\hline $7 / \mathrm{BH}-1 / \mathrm{BG}$ & 1474 & 0,1637 & 0,003593 & 76,79 \\
\hline
\end{tabular}

Observou-se que os animais de primeira lactação apresentaram menores valores dos parâmetros por toda a lactação, ou seja, menor produção inicial, menor taxa de acréscimo até o pico e menor produção no pico. Alguns autores também reportaram estes menores valores para animais de primeira lactação em estudos de curvas de lactação (QUEIROZ et al., 1991 e JUNQUEIRA et al., 1997, com vacas holandesas;
RIBEIRO \& PIMENTA FILHO, 1999, com cabras mestiças; $\mathrm{COBUCl}$ et al., 2001, com vacas Guzerá).

$\mathrm{Na}$ Tabela 3 estão os valores de produção inicial, produção de leite no pico de lactação, tempo para atingir o pico e persistência na lactação, calculados pelo modelo de Wood (1967) ajustado por metodologia bayesiana.

\begin{tabular}{|c|c|c|c|c|}
\hline \multirow{2}{*}{$\begin{array}{c}\text { Gropo } \\
\text { generico }\end{array}$} & $\begin{array}{l}\text { Producaso } \\
\text { inicisl }(\mathrm{kg})\end{array}$ & $\begin{array}{l}\text { Produçao no } \\
\text { pico (kg) }\end{array}$ & $\begin{array}{c}\text { Tempo para atingir o } \\
\text { pico (dias) }\end{array}$ & $\begin{array}{l}\text { Persisten } \\
\text { eia }\end{array}$ \\
\hline & \multicolumn{4}{|c|}{$T^{*}$ lastapsa } \\
\hline $1,4 \mathrm{H}-3 / 4 \mathrm{G}$ & 8.27 & 11.02 & 39,76 & 8.550 \\
\hline $1 / 2 \mathrm{H}-1 / 2 \mathrm{G}$ & 11,28 & 16.82 & 44,94 & 6.574 \\
\hline S.BH-3/EG & 11,19 & 15,40 & $3 \mathrm{t}, 54$ & 8,463 \\
\hline $3,4 \mathrm{H}-1 / 4 \mathrm{~S}$ & 10.89 & 17.13 & 49,49 & 6.658 \\
\hline \multirow[t]{2}{*}{$78 \mathrm{H}-160$} & 13,30 & 21.39 & 50,46 & 6.688 \\
\hline & \multicolumn{4}{|c|}{$2^{4}$ betapso } \\
\hline $1 / 4 \mathrm{H}-3 / 4 \mathrm{G}$ & 11,22 & 14.49 & 34,25 & 6,415 \\
\hline $1 / 2 \mathrm{H}-1 / 2 \mathrm{G}$ & 14,05 & 18,91 & 34,77 & 6,362 \\
\hline $5,8 \mathrm{H}-3 / 83$ & 12,67 & 18,48 & 37,63 & 6,368 \\
\hline $3,4 \mathrm{H}-1 / 4 \mathrm{G}$ & 14,49 & 20.16 & 37.49 & 6.414 \\
\hline $7 / 8 \mathrm{H}-1 / 80$ & 14,74 & 23.38 & 45,56 & 6.550 \\
\hline
\end{tabular}

Os animais de primeira lactação demoraram mais tempo para atingir 0 pico de produção e apresentaram maiores valores de persistência na lactação.

Segundo Cobuci et al., (2003), o parâmetro "c" dos modelos utilizados no estudo de curvas de lactação pode ser considerado como medida de persistência, uma vez que está associado à taxa de declínio da produção de leite após o pico. Porém, comparar a persistência entre animais apenas por este parâmetro não seria ideal, pois a persistência tem relação com a produção no pico e o tempo para atingir este pico. Em alguns desses 
modelos, a persistência na lactação pode ser obtida pela combinação de parâmetros específicos do modelo, a exemplo da medida de persistência na lactação fornecida pelo modelo de Wood (1967): persistência = $[-$ $(b+1) \ln (c)]$. De acordo com El Faro \& Albuquerque (2002) a persistência é um parâmetro que não tem unidade e seus maiores valores absolutos indicam maior persistência, o que permitiria uma comparação direta entre animais de interesse.

Em relação à maior persistência das fêmeas primíparas, Cobuci et al. (2001) verificaram que vacas da raça Guzerá de primeira ordem de parto se mostraram mais persistentes que as matrizes das demais ordens. Menor persistência em matrizes de ordens de partos mais avançadas foi verificada também por Rebouças et al. (2008), para vacas da raça Gir, e Glória et al. (2010), para vacas mestiças HolandêsZebu.

A menor produção de leite durante as fases da curva da lactação observada nas fêmeas primíparas está relacionada à menor diferenciação e menor população das células secretoras na glândula mamária destes animais. Esta menor produção também é observada na taxa de declínio dos animais desta ordem de parto, o que
Ihes confere maior persistência. Em virtude do menor desenvolvimento da glândula mamária, estes animais demoram mais tempo para atingir o pico de produção. Miller et al., (2006) observaram que a glândula mamária de vacas multíparas foi metabolicamente mais ativa do que de primíparas, especialmente no início e no pico de lactação, conferindo maior produção destes animais nestas duas fases da curva. De acordo com Sanders (1923), o aumento da ordem de lactação das vacas proporciona um aumento na produção de leite, porém, animais de primeira lactação se apresentam mais persistentes. Isso pode ser devido às vacas de primeira lactação estar ainda em fase de crescimento corporal e de desenvolvimento da glândula mamária e, portanto, teriam menor capacidade produtiva (SANTOS \& FONSECA, 2006). Vacas adultas podem produzir $20-30 \%$ a mais de leite do que vacas primíparas (BLOCK et al., 1995; SOUZA et al., 2010).

Considerando-se a Tabela 3 e as Figuras 1 e 2 verifica-se menor produção inicial, no pico e menor declínio de produção das fêmeas primíparas.

Comparando-se as ordens de lactação, foi observado que os animais de primeira ordem demoraram mais tempo 
para atingir o pico e, entre os grupos genéticos, este fato foi observado nos animais $7 / 8 \mathrm{H}-1 / 8 \mathrm{G}$; estas duas classes foram as que se mostraram mais persistentes (Tabela 3). Este fato pode ser explicado pela alta e positiva correlação entre os dias para chegar ao pico de produção e persistência da lactação: Tekerli et al., (2000) e Albarran-Portillo \& Pollott (2011) relataram valores de 0,80 e 0,64 , respectivamente, 0 que denota a estreita relação entre essas duas características, concluindo que picos de produção que ocorrem mais tardiamente estão associados a lactações mais persistentes.

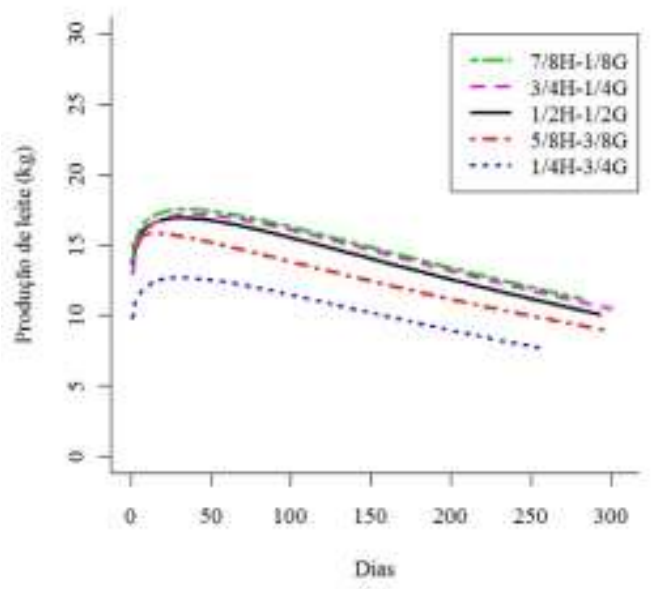

Figura 1 - Curvas de lactaça ào dos diferentes grupos genéticos. de primeira ordem, ajustadas pelo modelo de Wood (1967) por metodologia bayesiana.

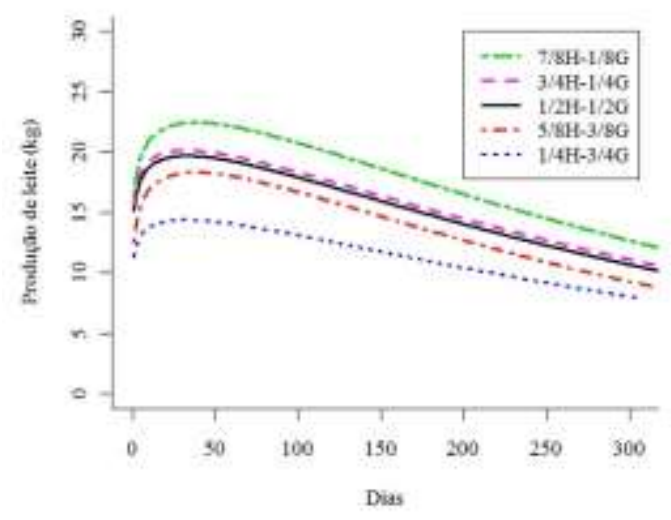

Figura 2 - Curvas de lactaçāo dos diferentes grupos genéticos, de segunda ordem, ajustadas pelo modelo de Wood (1967) por metodologia bayesiana.

Por outro lado, associação negativa $(-0,22)$ entre pico de produção e persistência da lactação também foi relatada (TEKERLY et al., 2000), ou seja, vacas que expressam elevada produção no pico tendem a apresentar maior taxa de decréscimo após a produção máxima. No caso do grupo 7/8H-1/8G, os animais apresentaram elevada produção inicial, elevada produção no pico e, também, alta taxa de declínio de produção após o pico, o que thes proporcionou manter elevada produção durante todas as fases da curva de lactação. Além disso, este grupo foi o que demorou mais tempo para atingir o pico de produção. Sendo assim, o fato de a produção ser elevada por toda a lactação aliado ao pico mais tardio, confere a estes animais maior persistência na lactação.

Animais mais persistentes estão sujeitos a um menor estresse fisiológico 
devido a menor produção no pico de lactação, o que minimiza a incidência de problemas reprodutivos e de doenças metabólicas (MADSEN, 1975; SÖLKNER \& FUCHS, 1987; GROSSMAN et al.,1999; TEKERLI et al., 2000). Além disso, vacas de maior persistência na lactação apresentam maior produção de leite e maior longevidade e podem ser consideradas produtoras mais eficientes, uma vez que fornecem retorno econômico, de certo modo, regular durante toda vida útil (LUDWICK \& PETERSEN, 1943). Vacas com curvas de lactação mais persistentes têm necessidades energéticas mais constantes por toda a lactação (DEKKERS et al., 1998).

$\mathrm{Na}$ Tabela 4 estão a duração de lactação e a produção total de leite para os diferentes grupos genéticos e duas ordens de parto. Foi possível observar que, dentro de cada ordem de parto, os grupos genéticos mostraram comportamento semelhante em relação à duração da lactação.
Tabela 4 - Duração da lactaçăo (DL) e produçảo total de leite (PTL) para vacas de

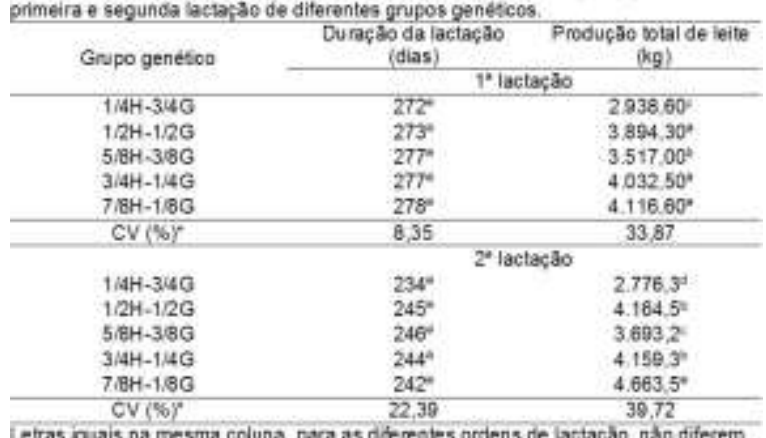
Letras guais na mesma coluna, para as diterentes ordens de lactaçác, nato difeentre si pelo leste de Tukey a $5 \%$ de probabilidade $(p>0,05$ ). CV $(\%)=$ coeficiente de variaçà

Não houve diferença no período de lactação dentro da mesma ordem de lactação, entretanto, entre ordens houve uma redução média de $12 \%$ da primeira para a segunda. Esses valores do período de lactação estão próximos aos observados por Barbosa et al., (1994), em Pernambuco, com vacas holandesas e de alta mestiçagem, por Madalena et al., (1996), em Minas Gerais, com vacas 1/4 HVB, e FACÓ et al., (2002), com dados de vacas Girolando, da Associação Brasileira dos Criadores de Girolando. Por outro lado, os valores ficaram abaixo dos relatados por Thaler Neto et al., (1996), com vacas Flamencas e Holandesas, em São Paulo, por Freitas et al., (2001), em Minas Gerais, com vacas holando-gir, de diferentes grupos genéticos, por Glória et al., (2006), em Brasília, com vacas Girolando, por McManus et al., (2008), em Brasília, com dados de vacas holandesas e mestiças holandogir, de diferentes grupos genéticos, e por Ribeiro et al., (2009), no Rio grande 
do Norte, com dados de vacas Gir e Guzerá. Um fato relevante a ser considerado foi a redução do período de lactação, da primeira para a segunda lactação, quando, normalmente, esperava-se aumentar, embora tenha ocorrido um aumento médio na produção de leite em torno de 5,2\%. Na literatura consultada não se observou nada semelhante, com exceção dos trabalhos de Ribas et al., (1983), no Paraná, e Thaler Neto et al., (1990), em Santa Catarina, quando relataram redução linear significativa do período de lactação de vacas holandesas PO e PC à medida que avançava a idade das vacas ao parto. Já Guimarães et al., (2002), trabalhando com animais das raças Gir, Holandês e mestiças HG não relataram efeito significativo das diferentes ordens de parto sobre 0 período de lactação das vacas. A princípio, é possível que este fato tenha respaldo no manejo dos animais, principalmente, alimentar, pois não raro os produtores propiciam melhores condições criatórias aos animais de primeira cria.

Quanto à produção de leite, houve diferença significativa entre os grupos genéticos avaliados, com tendência de aumento na produção à medida que aumentava a quantidade de genes da raça especializada, indistintamente da ordem de lactação. Portanto, as menores produções foram observadas nos animais $1 / 4 \mathrm{HG}$ e as maiores produções nos animais $7 / 8$ HG. Exceção à regra é o que aconteceu com os animas $1 / 2 \mathrm{HG}$, em que na primeira lactação esses animais foram tão produtivos $(P>0,05)$ quanto os $7 / 8 \mathrm{HG}$, e na segunda lactação, em que eles foram inferiores apenas em relação aos animais $7 / 8$ HG. Provavelmente, essa maior produção de leite nesse grupamento genético seja consequência da heterose, uma vez que a maior expressão desse fenômeno ocorre logo na primeira geração (BARBOSA et al., 2008). McManus et al., (2008) também relataram maiores produções de leite, em Brasília, para vacas 1/2 HG, acompanhadas das produções de vacas $3 / 4$ HG. Por outro lado, Freitas et al., (2001), trabalhando com lactações de vacas holando-zebu, de diferentes grupos genéticos, em Minas Gerais, constataram que as mais baixas produções ocorreram nos animais $1 / 2$ $\mathrm{HZ}$.

Os valores médios de produção de leite observados (Tabela 4) são superiores aos relatados por McManus et al., (2008), em Brasília, com animais holandeses e mestiços holando-gir, e por Facó et al., (2002), com dados de vacas Girolando, de diferentes grupos 
genéticos, da Associação Brasileira dos

Criadores de Girolando. Por outro lado, as médias de produção aqui encontradas são inferiores aquelas relatadas por Freitas et al., (2001), com animais de variados grupos genéticos ( $1 / 2 \mathrm{HZ}$ até $31 / 32 \mathrm{HZ}$ ), em Minas Gerais, e por Glória et al., (2006), com animais $1 / 2,3 / 4$ e $7 / 8$ HG, em Brasília. De modo geral, os trabalhos evidenciam aumento na produção de leite, provocado pela maior proporção de genes da raça especializada, como se constatou nesse trabalho, revezando entre animais $3 / 4 \mathrm{e}$ $7 / 8 \mathrm{HG}$, entretanto, em função de maior ou menor virtuose promovida pela heterose é possível que em algumas situações o grupo genético $1 / 2 \mathrm{HG}$ seja 0 mais indicado.

\section{CONCLUSÃO}

A abordagem bayesiana no ajuste do modelo de Wood permitiu estimativas acuradas das características da curva de lactação dos animais, da produção de leite e da duração e persistência da lactação.

Animais de primeira lactação apresentaram valores inferiores de produção de leite durante as fases da curva de lactação, quando comparados aos de segunda ordem de lactação, embora sejam mais persistentes.

\section{AGRADECIMENTOS}

À EMBRAPA/CNPGL pela cessão dos dados.

\section{REFERÊNCIAS}

ALBARRÁN-PORTILLO, B.; POLLOTT, G.E. Environmental factors affecting lactation curve parameters in the United Kingdom's commercial dairy herds. Archivos de Medicina Veterinaria, v. 43, p. 145-153, 2011.

BARBOSA, S.B.P.; RAMALHO, R.P.; MONARDES, H.G.; DIAS, F.M.; SANTOS, D.C.; BATISTA, A.M.V. Milk and fat production of crossbred HolsteinGir cows (Bos Taurus taurus-Bos taurus indicus) in the Agreste region of the Brazilian state of Pernambuco. Genetics and Molecular Biology, v. 31, n. 2, p.468-474, 2008.

BATRA, T.R. Comparation of two mathematical models in fitting lactation curves for pureline and crossline dairy cows. Canadian Journal of Animal Science, v.66, n.2, p.405 414, 1986.

BLOCK, E.; BURCHARD, J.F.; MONARDES, H.G. Manual do curso de bovinos leiteiros. Montreal: McGill University, 1995.

COBUCI, J.A.; EUCLYDES, R.F.; TEODORO, R.L. et al. Aspectos genéticos e ambientais da curva de lactação de vacas da raça Guzerá. Revista Brasileira de Zootecnia, v. 30, n 4, p. $1204-1211,2001$.

COBUCl, J.A. Uso de modelos de regressão aleatória na avaliação da persistência de animais da raça Holandesa. 99p.Tese (Doutorado) Universidade Federal de Viçosa, 2002.

COBUCl, J.A.; EUCLYDES, R.F.; PEREIRA, C.S. et al. Persitência na lactação - uma revisão. Archivos 
Latinoamericnos de Produción Animal, v.11, n. 3, p.163-173, 2003.

COBUCI, J.A.; EUCLYDES, R.F.; COSTA, C.N. et al. Análise de persistência na lactação de vacas da raça Holandesa, usando produção no dia do controle e modelo de regressão aleatória. Revista Brasileira de Zootecnia, v. 33, n. 3, p. 546-554, 2004. COSTA, C.N.; MILAGRES, J.C.; CASTRO, A.C.G. et al. Efeitos da estação e idade ao parto sobre a curva de lactação de vacas da raça holandesa. Revista Brasileira de Zootecnia, v.11, n.2, p.320-330, 1982.

DEKKERS, J.C.M.; TEM HAG, J.H.; WEERSINK, A. Economic aspects of persistency of lactation in dairy cattle. Livestock Production Science, v.53, p.237-252, 1998.

EL FARO, L.; ALBUQUERQUE, L.G. Comparação de alguns modelos matemáticos para o ajuste às curvas de lactação individuais de vacas da raça Caracu. Arquivo Brasileiro de Medicina Veterinária e Zootecnia, v.54, n.3, p.295-302, 2002.

FACÓ, O.; LÔBO, R.N.B.; MARTINS FILHO,R. et al. Análise do Desempenho Produtivo de Diversos Grupos Genéticos Holandês x Gir no Brasil. Revista Brasileira de Zootecnia, v.31, n.5, 1944-1952, 2002.

FREITAS, M.S.; DURÃES, M.C.; FREITAS, A.F. et al. Comparação da produção de leite e de gordura e da duração da lactação entre cinco "graus de sangue" originados de cruzamentos entre Holandês e Gir em Minas Gerais. Arquivo Brasileiro de Medicina Veterinária e Zootecnia, v.53, n.6, p.708-713, 2001.

GLÓRIA, J.R.; BERGMANN, J.A.G.; REIS, R.B. et al. Efeito da composição genética e de fatores de meio sobre a produção de leite, a duração da lactação e a produção de leite por dia de intervalo de partos de vacas mestiças Holandês-Gir. Arquivo Brasileiro de Medicina Veterinária e Zootecnia, v.58, p.1139-1148, 2006.

GLÓRIA, J.R.; BERGMANN, J.A.G.; QUIRINO, C.R. et al. Curvas de lactação de quatro grupos genéticos de mestiças Holandês-Zebu. Revista Brasileira de Zootecnia, v.39, n.10, p.2160-2165, 2010.

GONÇALVES, T.M.; MARTINEZ, M.L.; MILAGRES, J.C. Curvas de lactação na raça Gir. I. Escolha do modelo de melhor ajuste. Revista Brasileira de Zootecnia, v. 25, n. 4, p. 616-626, 1996.

GROENEWALD, P.C.N.; VILJOEN, C.S. $A$ Bayesian model for analysis of lactation curves of dairy goats. Journal of Agricultural, Biological and Environmental Statistics, v.8, p.75-262 83, 2003.

GROSSMAN, M.; HARTZ, S.M.; KOOPS, W.P. Persistency of lactation yield: A novel approach. Journal of Dairy Science, v. 82 , n. 10, p. 21922197, 1999.

GUIMARÃES, J.D.; ALVES, N.G.; COSTA, E.P. et al. Eficiências reprodutiva e produtiva em vacas das raças Gir, Holandês e cruzadas Holandês x Zebu. Revista Brasileira de Zootecnia, v.31, n.2, p.641-647, 2002.

HEIDELBERGER, P.; WELCH, P.D. Simulation run length control in the presence of an initial transient. Operations Research, v.31, n.6, p.1109-1144, 1983.

JUNQUEIRA, L.V.; NEIVA, R.S.; VEIGA, R.D. et al. Estudo das curvas de lactação de vacas Holandesas de alguns rebanhos do Estado de Minas Gerais, por intermédio de uma função gama incompleta. Revista Brasileira de Zootecnia, v. 26, n.6, p.1109-1118, 1997.

LUDWICK, T.M.; PETERSEN, W.E. A measure of persistency of lactation of 
dairy cattle. Journal of Dairy Science, v. 26, p. 439-445, 1943.

MADALENA, F.E.; LEMOS, A.M.; TEODORO, R.L. et al. Desempenho comparativo de vacas mestiças de Holandês com Guzerá de origem leiteira e não leiteira. In: REUNIÃO ANUAL DA SOCIEDADE BRASILEIRA DE ZOOTECNIA, 33., 1996, Fortaleza. Anais... Fortaleza: Sociedade Brasileira de Zootecnia, 1996. p.3-4.

MADSEN, O. A comparison of some suggested measures of persistency of milk yield in dairy cows. Animal Production, v. 20, p. 191-197, 1975.

McMANUS,C.; TEIXEIRA, R.A.; DIAS, L.T. et al. Características produtivas e reprodutivas de vacas Holandesas e mestiças Holandês $\times$ Gir no Planalto Central. Revista Brasileira de Zootecnia, v.37, n.5, p.819-823, 2008.

MILLER, R.H.; MCDANIEL, B.T.; CORLEY, E.L. Variation in ratio factors for age- adjusting part-lactation records. Journal of Dairy Science, v. 50, n.11, p. 1819-1823, 1967.

MILLER, N.; DELBECCHI, L.; PETITCLERC, D. et al. Effect of stage of lactation and parity on mammary gland cell renewal. Journal of Dairy Science, v. 89, p. 4669-4677, 2006.

QUEIROZ, S.A.; FREITAS, M.A.R.; ALBUQUERQUE, L.G. et al. Fatores genéticos e de meio que influenciam os componentes da curva de lactação de bovinos da raça Holandesa. Arquivo Brasileiro de Medicina Veterinária e Zootecnia, v. 43, n.4, p. 357-370, 1991.

R Development Core Team (2012). R: A language and environment for statistical computing. R Foundation for Statistical Computing, Vienna, Austria. ISBN3900051-07-0, URL http://www.Rproject.org.

REBOUÇAS, G. F.; GONÇALVES, T. M.; MARTINEZ, M. L. et al. Novas funções para estimar a produção de leite, em 305 dias de lactação, de vacas da raça Gir. Revista Brasileira de Zootecnia, v.37, n.7, p. 1222-1229, 2008.

REKAYA, R.; CARABÃNO, M.J.; TORO, M.A. Bayesian analysis of lactation curves of Holstein-Friesian cattle using a nonlinear model. Journal of Dairy Science, v.83, p.2691-2701, 2000.

RIBEIRO, M.N.; PIMENTA FILHO, E.C. Estudo de Efeitos Ambientais que Influem na Forma da Curva de Lactação de Cabras Mestiças no Estado da Paraíba. Revista Brasileira de Zootecnia, v. 28, n.4, p. 868-874, 1999.

SANDERS, H.G. The shape of lactation curve. Journal of Agricultural Science, v.13, p. 169-179, 1923.

SANTOS, M.V.; FONSECA, L.F.L. Estratégias para Controle de Mastite e Melhoria da Qualidade do Leite. 1.ed. Barueri: Editora Manole, 2006. $314 p$.

SILVA, F.F.; MUNIZ, J.A.; AQUINO, L.H. et al. Análise Bayesiana da curva de lactação de cabras Saanen. Pesquisa Agropecuária Brasileira, v. 40, n. 1, p. 27-33, 2005.

SILVA, M.V.G.; PAIVA, L.C.; CEMBRANELLI, M.A.R. et al. Programa de Melhoramento Genético da Raça Girolando - Teste de Progênie: Sumário de Touros 2011. - Juiz de Fora: Embrapa Gado de Leite, 2011. 46p. (Documentos, 148).

SÖLKNER, J.; FUCHS, W. A comparison of different measures of persistency with special respect to variation of Test-day milk yields. Livestock Production Science, v. 16, p.305-319, 1987.

SOUZA, R; SANTOS, G.T.; VALLOTO, A.A. et al. Produção e qualidade do leite de vacas da raça Holandesa em função da estação do ano e ordem de parto. Revista Brasileira de Saúde e 
Produção Animal, v. 11, n. 2, p. 484495, 2010.

TEKERLI, M.; AKINCI, Z.; DOGAN, I. et al. Factors affecting the shape of lactation curves of Holstein cows from the Balikesir province of Tukey. Journal of Dairy Science, v. 83, n.6, p.13811386, 2000.

TEODORO, R.L.; VERNEQUE, R.S. Sistema de cruzamento como alternativa para o melhoramento de bovinos. In: REUNIÃO ANUAL DA SOCIEDADE BRASILEIRA DE ZOOTECNIA, 36. 1999, Porto Alegre. Anais... Porto Alegre: Sociedade Brasileira de Zootecnia, 1999. p.127141.

THALER NETO, A.; MÜHLBAUER, M.D.; ZARDO, W.F. et al. Fatores que afetam a produção de leite e o período de lactação em um rebanho das raças Flamenga e Holandesa no planalto catarinense. Ciência Rural, v.26, n.1, p.123-128, 1996.

WOOD, P.D.P. Algebraic model of the lactation curve in cattle. Nature. v. 216, p. 164-165, 1967. 